\title{
Erratum to: Multi-loop Damping and Tracking Strategy Emulating a Butterworth Pattern for Accurate Nanopositioning
}

\author{
Mohammed Altaher and Sumeet S. Aphale ${ }^{(\bowtie)}$ \\ University of Aberdeen, Aberdeen, UK \\ \{mohammed. altaher, s.aphale\}@abdn. ac.uk
}

\section{Erratum to:}

Chapter "Multi-loop Damping and Tracking Strategy Emulating a Butterworth Pattern for Accurate Nanopositioning" in: M.S. Mohamed Ali et al. (Eds.), Modeling, Design and Simulation of Systems, Part I, CCIS 751, https://doi.org/10.1007/978-981-10-6463-0_2

The initially published versions of Figs. 4 and 5 are incorrect. These are the correct versions:

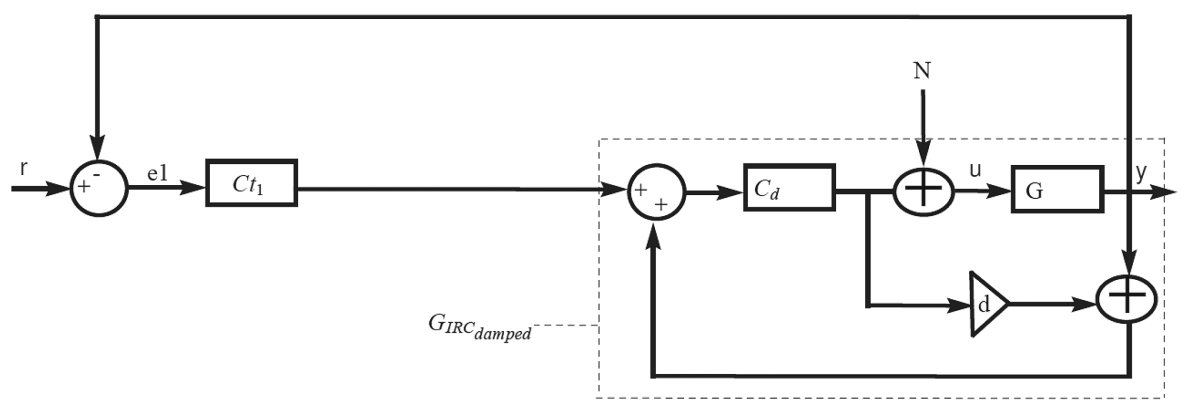

Fig. 4. Schematic of the traditional damping + tracking control scheme

The updated online version of this chapter can be found at https://doi.org/10.1007/978-981-10-6463-0_2 


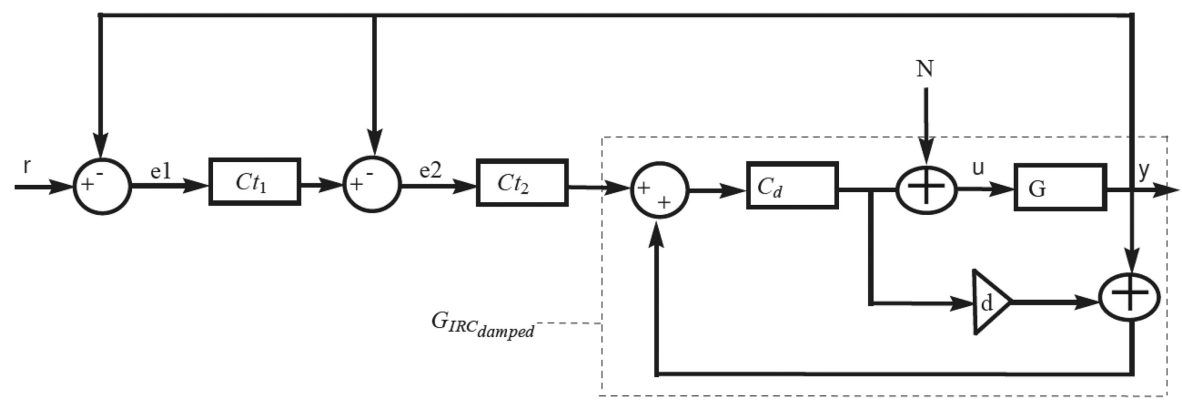

Fig. 5. Schematic of the proposed control scheme with dual-loop tracking + damping 